Cahiers $d u$ MONDE RUSSE

\section{Cahiers du monde russe}

Russie - Empire russe - Union soviétique et États indépendants

$50 / 2-3 \mid 2009$

L'Europe orientale, 1650-1730. Crises, conflits et renouveau

\title{
Magnats et noblesse de Lituanie face à l'empire russe (1700-1730)
}

Lithuanian nobility's and magnates' dealings with the Russian Empire

(1700-1730)

\section{Andrej Macuk}

\section{OpenEdition \\ Journals}

Édition électronique

URL : https://journals.openedition.org/monderusse/9726

DOI : $10.4000 /$ monderusse. 9726

ISSN : $1777-5388$

Éditeur

Éditions de l'EHESS

Édition imprimée

Date de publication : 15 septembre 2009

Pagination : 495-510

ISBN : 978-2-7132-2260-3

ISSN : $1252-6576$

\section{Référence électronique}

Andrej Macuk, « Magnats et noblesse de Lituanie face à l'empire russe (1700-1730) », Cahiers du

monde russe [En ligne], 50/2-3 | 2009, mis en ligne le 13 octobre 2012, consulté le 03 septembre 2022 URL : http://journals.openedition.org/monderusse/9726; DOI : https://doi.org/10.4000/monderusse. 9726 


\section{MAGNATS ET NOBLESSE DE LITUANIE FACE À L'EMPIRE RUSSE (1700-1730)}

La seconde Guerre du Nord (1700-1721) a marqué un tournant dans l'histoire de la Russie et de la République'. Tandis que la Russie s'impose comme puissance militaire considérable, avec l'avis de laquelle il faut désormais compter sur la scène internationale, capable aussi d'influer sur la situation politique intérieure de la Suède comme de la République, cette dernière se trouve confrontée à une crise politique, interne justement, de grande ampleur. Simultanément, elle perd beaucoup de son importance dans les affaires internationales. Des changements significatifs interviennent, de même, dans l'attitude de l'élite du grand-duché de Lituanie (ci-après : GDL) vis-à-vis de la Russie, attitude qui passe, selon l'historien lituanien Gintautas Sliesoriūnas, « de la mégalomanie à la servilité »².

Pourtant, au tout début du XVIII ${ }^{\mathrm{e}}$ siècle, la Russie et la République sont alliées. Dans les moments difficiles qui marquent le début de la seconde Guerre du Nord, l'alliance tient bon, en grande partie grâce à l'attitude des principaux lignages de magnats du GDL : les Pociej, les Ogiński, les Wiśniowiecki. Ils s'en tenaient à la

1. Rzeczpospolita (Polska) - abréviation de Rzeczpospolita Obojga Narodów, Polskiego i Litewskiego [République des Deux Nations, polonaise et lituanienne] - État fédératif résultant de l'Union de Lublin (1569) entre le royaume de Pologne et le grand-duché de Lituanie. Celui-ci conserve son nom, cependant que les territoires du royaume sont dits « de la Couronne ». Le terme juridique Korona Królestwa Polskiego (Corona Regni Poloniae) [la Couronne du royaume de Pologne] date du XIV ${ }^{\mathrm{e}}$ siècle. Il précise le statut de l'État [la Couronne] dans ses rapports à la personne du souverain. Une séparation de ces deux entités, distinctes tant du point de vue politique que du droit, est instituée. Ce système garantit essentiellement l'inaliénabilité du territoire (Korona) dont le souverain ne peut rien distraire à son gré. (NdT)

2. Sliesoriūnas Gintautas, "Changes in Attitudes towards Russia among the LithuanianPolish Elite at the Turn of the Seventeenth and Eighteenth Centuries », Lithuanian Historical Studies, 9, 2004, p. 1 ; Vladimir Alekseevič Artamonov, Rossija i Reč Pospolitaja posle Poltavskoj pobedy [La Russie et la République après la victoire de Poltava], M., 1990, p. 25 ; Vladimir Dorofeevič Koroljuk, « Vstuplenie Reči Pospolitoj v Severnuju vojnu » [ L'entrée de la République dans la Guerre du Nord »], Učënye zapiski Instituta slavjanovedenija, 10, 1954, p. 240-271 ; Andrzej Kamiński, « Początki antyszwedzkiego sojuszu : Ze stosunków polsko-rosyjskich 1704-1706 » [« Les prémisses de l'alliance antisuédoise. Aspects des relations polono-russes, 1704-1706 »], Przegląd Historyczny, 60, 2, 1969, p. 291-313. 
vision traditionnelle de la Russie partagée par une partie des magnats du GDL : la Russie était une alliée de poids dans les conflits internes de la République. Le 16 avril 1702, ils concluent au nom du GDL, dénommé dans le texte Reč Pospolitaja Litovskaja [République lituanienne], une alliance séparée avec Pierre I ${ }^{\mathrm{er}}$. La position des Pociej, des Ogiński et des Wiśniowiecki en faveur de cette alliance, contractée en dehors de la Couronne, s'explique dans une large mesure par le conflit qui les oppose au sein du GDL aux Sapieha, soutenus, de leur côté, par le roi de Suède Charles XII.

Après sa victoire de Poltava le 8 juillet 1709 , Pierre I ${ }^{\text {er }}$ n'oublie pas ses alliés du GDL, et contraint de fait le roi Auguste II à nommer comme nouveau grand hetman ${ }^{3}$ du GDL Ludwik Pociej. À partir de cette date et jusqu'à sa mort en 1730, le nouveau grand hetman devient l'allié principal du tsar au sein du grand-duché, bénéficiant du soutien de Pierre ${ }^{\text {er }}$ dans tous ses conflits avec Auguste II. Qui plus est, certaines démarches de Pociej dirigées contre le roi étaient en fait suggérées par le tsar.

L'un des problèmes clés auquel la société polonaise devait faire face, pendant la seconde Guerre du Nord, était celui de la réforme de l'État. Il ne fait pas de doute que le roi, la noblesse et les magnats ne partageaient pas, sur la question, le même point de vue. Leurs divergences apparaissent de manière flagrante lors de la formation des confédérations de la noblesse : celle de Tarnogród dans les territoires de la Couronne en 1714 et celle de Wilno dans le GDL l'année suivante. La réforme en profondeur des institutions de la République n'en est que plus urgente, et ces dissensions affectent en outre la relation de la noblesse et des magnats envers la Russie.

Pierre $\mathrm{I}^{\text {er }}$ est opposé à un renforcement du pouvoir monarchique dans la République et soutient pour cette raison les assauts de la noblesse contre le roi. La situation change cependant avec la formation de la confédération de Tarnogród. La noblesse en effet n'intervient pas seulement contre le roi, mais aussi contre les soutiens russes du hetman. La Russie réussit à jouer le rôle de médiatrice dans la réconciliation entre la noblesse et le roi $^{4}$, dont les principaux points sont inclus dans le Traité de Varsovie de 1716 et confirmés par la Diète de 1717. En dépit de tous ses efforts, cependant, la Russie n'arrive pas, du fait des dispositions antirusses de la noblesse, à prendre une part active dans l'élaboration des conditions de la réconciliation. De ce fait, elle ne peut s'opposer à l'inclusion dans l'accord final de clauses limitant le pouvoir du hetman ${ }^{5}$.

3. On compte à cette époque quatre hetmans, commandants en chef de l'armée après le roi : deux hetmans de la Couronne, le grand hetman et son subordonné, le hetman de camp, et deux hetmans du GDL qui portent les mêmes titres que ceux de la Couronne. (NdT)

4. Józef Andrzej Gierowski, «Wokół mediacji w traktacie warszawskim » [« Autour de la médiation lors du traité de Varsovie »], Zeszyty Naukowe Uniwersytetu Jagiellońskiego : Prace Historyczne, 26, 1969, p. 58.

5. J.A. Gierowski, « "Opisanie” urzędów centralnych przez konfederatów tarnogrodzkich » [ «a "description" des organes centraux de gouvernement par les confédérés de Tarnogród»], in $O$ naprawę Rzeczypospolitej XVII-XVIII w. Prace ofiarowane Władysławowi Czaplińskiemu W 60 rocznicę urodzin [La réforme de la République, $X V I I^{e}-X V I I I^{e}$ siècle. Travaux offerts à Władysław Czapliński pour son $60^{\mathrm{e}}$ anniversaire],Varsovie : Państwowe Wydawnictwo Naukowe, 1965, p. 207. 
La Russie ne parvient pas davantage à se faire désigner comme garante de l'application du Traité de Varsovie ni des décisions de la Diète de $1717^{6}$. Certes, le gouvernement russe ne manquera pas, par la suite, de se prévaloir de son rôle de médiateur pour se poser en garant des institutions de la République. Cette manière d'agir est rendue possible par la politique d'opposition au roi des magnats du GDL, qui appellent régulièrement au secours leur voisine orientale. Cependant, l'action de la confédération de Tarnogród met au jour les divergences entre la noblesse et les magnats quant à la perception de la Russie et de son intervention dans les affaires intérieures de la République. Si la noblesse s'oppose à toute interférence de la Russie, les hetmans, par contre, représentants des magnats, sont à l'origine de l'interventionnisme russe.

Quant à la Cour, elle voit dans l'intervention russe une tentative de contrecarrer les projets d'Auguste II tendant à renforcer le pouvoir royal et instaurer une monarchie héréditaire au profit de la maison saxonne des Wettin. En ce qui concerne le GDL, la Cour s'efforce de faire aboutir ses plans à l'aide d'une faction nouvellement formée qui lui est favorable, et qui est constituée principalement des partisans du groupe aristocratique des Czartoryski ${ }^{7}$. L'opposition antimonarchique est emmenée par les hetmans. Leurs contacts avec Pierre $\mathrm{I}^{\mathrm{er}}$ remontent à l'époque de la seconde Guerre du Nord, et ils continuent de voir en la Russie le plus sûr rempart de leurs prérogatives contre le roi et contre la noblesse. Lorsque les relations se tendent entre Saint-Pétersbourg et Varsovie et que la République se montre tentée de rejoindre l'Union de Vienne, les hetmans coordonnent leur action avec celle du tsar par l'intermédiaire des ambassadeurs russes auprès de la République ${ }^{8}$. À plusieurs reprises, les hetmans exigent le retour sous leur autorité des « troupes sur le modèle étranger » (wojskaautoramentu cudzoziemskiego) et sont soutenus par la Russie, qui voit là une occasion supplémentaire de rogner le pouvoir monarchique et d'entraver l'adhésion de la République à l'Union de Vienne. Ce sont les hetmans eux-mêmes qui cherchent alors à tirer profit du soutien russe pour renforcer leurs positions, mais l'hostilité de leurs propres partisans envers la Russie limite leur marge de manœuvre 9 . Ceux qui comptent alors le plus sur le soutien russe sont le

6. Gierowski, « Wokół mediacji... », p. 67.

7. Urszula Kosińska, « Początki kariery Stanisława Poniatowskiego w służbie Augusta II » [ « Les débuts de la carrière de Stanisław Poniatowski au service d'Auguste II »], in Mariusz Markiewicz, Ryszard Skowron, éds., Faworyci i opozycjoniści : Król a elity polityczne w Rzeczypospolitej $X V$-XVIII wieku [Favoris et opposants (Le roi et les élites politiques de la République, $X V^{e}-X V I I I^{e}$ siècle)], Cracovie : Zamek Królewski na Wawelu, 2006, p. 417.

8. U. Kosińska, Sejm 1719-1720 a sprawa ratyfikacji traktatu wiedeńskiego [La Diète de 17191720 et la question de la ratification du traité de Vienne], Varsovie : Semper, 2003,p. 207-208, 242-244.

9. Tomasz Ciesielski, «Zabiegi hetmanów o rewizję uchwał sejmu niemiego i odzyskanie komendy nad autoramentem cudzoziemskim a sejmy w latach 1717-1724 (prolegomena) » [ «Les tentatives des hetmans pour obtenir la révision des décisions de la Diète muette, retrouver le commandement des troupes étrangères, et les Diètes des années 1717-1724 (introduction) »], in J. Muszyńska, éd., Rzeczpospolita w dobie wielkiej wojny północnej [La République à l'époque de la seconde Guerre du Nord], Kielce : Wydawnictwo Akademii Świętokrzyskiej, 2001, p. 69. 
grand hetman Ludwik Pociej et l'hetman de camp du GDL Stanisław Denhoff ${ }^{10}$. Ce dernier, avec l'aide de la Russie, va jusqu'à tenter de former dans le GDL, en 1719, une confédération antimonarchique dont l'un des buts est de détrôner Auguste II ${ }^{11}$.

La mort de Pierre ${ }^{\mathrm{er}}$ ne modifie pas substantiellement les relations entre Ludwik Pociej et le gouvernement russe. Cela alors que l'influence de la France se renforce au GDL : en 1729, Pociej consent même à soutenir la candidature de Stanisław Leszczyński au trône de Pologne-Lituanie, mais à condition que la Russie soit $\mathrm{d}^{\text {'accord }}{ }^{12}$. Une telle position peut paraître d'autant plus surprenante que le grand hetman du GDL s'était jusqu'alors signalé par son habileté à moduler son soutien en fonction de la conjoncture. Elle s'explique, en fait, par la conviction de Pociej que la Russie constitue une force capable d'intervenir dans les affaires intérieures de la République, opinion qui doit beaucoup à ses contacts avec l'élite politique russe : Pierre I ${ }^{\text {er }}$, mais aussi Aleksandr Menšikov, Grigorij Dolgorukij, et al.

Le revirement le plus frappant à l'égard de la Russie est celui de Jan Kazimierz Sapieha, partisan des Suédois et grand hetman du GDL nommé par Stanisław Leszczyński, qui, dès 1719-1720, devient l'un des soutiens les plus actifs de la Russie $^{13}$. Les Sapieha cependant ne reçoivent pas aussitôt l'appui de la Russie contre Auguste II, à cause du conflit entre Jan Kazimierz et les chefs du groupe prorusse dans le GDL, Ludwik Pociej et Stanisław Denhoff, au sujet des nominations qui dépendaient des hetmans. Et ce n'est qu'après que Pociej et Denhoff eurent donné leur accord que Sapieha recevra l'aide de la Russie ${ }^{14}$.

\section{Sapieha}

Sapieha, habile général et homme d'État expérimenté, a exercé, entre 1725 et 1730, une influence déterminante sur tous les anciens partisans de Stanisław Leszczyński, qu'il convertit aux idées prorusses. Son « système » en matière de politique étrangère et la façon dont il l'a mis en œuvre n'ont jamais été étudiés jusqu'ici. Une analyse de ses relations avec l'élite politique russe, en premier lieu avec Aleksandr Menšikov, est pourtant indispensable pour comprendre comment il en est arrivé à envisager le retour de Stanisław Leszczyński sur le trône de la République, qui plus est avec le soutien russe. Il faut pour cela explorer le monde de Jan Kazimierz Sapieha : qui sont ses alliés, qui ses ennemis dans le GDL et en Russie ? Quelle sorte d'homme est-il ? Dans quelle mesure son action personnelle influence-t-elle

10. Ibid., p. 67, 69. Stanisław Michał Ernest Denhoff (lit. Stanislovas Denhofas, all. Stanislaus Denhoff, ou Dönhoff).

11. Urszula Kosińska, « Rosyjskie plany wywołania antykrólewskiej konfederacji i detronizacji Augusta II w 1719 r. » [«Les plans de la Russie en 1719 pour la formation d'une confédération et pour détrôner Auguste II »], Kwartalnik historyczny, 3, 1999, p. 56.

12. Polski Słownik Biograficzny [Dictionnaire biographique polonais], 27, 1, 1982, p. 45.

13. Kosińska, « Rosyjskie plany wywołania... », p. 65.

14. Ibid., p. 55. 
le cours des événements? La question est d'autant plus intéressante que les Sapieha sont, de tous les magnats du GDL, ceux qui se sont le mieux intégrés dans l'élite dirigeante de Russie. De sorte que c'est la biographie et le système de représentations d'un homme d'État qui deviennent ici notre objet d'étude.

En 1720, Jan Kazimierz envoie son fils Piotr à Saint-Pétersbourg ${ }^{15}$, où ce dernier devient pour de longues années l'hôte du palais d'Aleksandr Menšikov ${ }^{16}$. C'est alors que sont formés les premiers projets de mariage entre Piotr Sapieha et la fille de Menšikov, Marija ${ }^{17}$. Pour Jan Kazimierz, une alliance matrimoniale avec les Menšikov constitue le meilleur gage d'un soutien russe aux projets des Sapieha dans le GDL. Il nous semble personnellement que le but principal des Sapieha était de recevoir les « possessions de Neuburg », données à bail depuis 1714 au roi Auguste II $^{18}$. En août-septembre 1720 , le tsar Pierre Ir lui-même s'était intéressé à l'avenir de ces possessions. La cour de Russie cherchait à intervenir dans cette affaire en soutenant les magnats du GDL qui lui étaient favorables. Menšikov, chargé des négociations, convia les hetmans et les Sapieha pour tenir un conseil secret dans sa propriété du GDL, Gorki ${ }^{19}$.

La mort de Pierre $\mathrm{I}^{\mathrm{er}}$ et l'avènement de Catherine $\mathrm{I}^{\mathrm{re}}$ renforcent la position de Menšikov. En 1725, il invite Jan Kazimierz Sapieha à Saint-Pétersbourg dans le but de discuter du mariage de sa fille avec Piotr, le fils de Jan Kazimierz. Menšikov compte sur l'alliance avec les Sapieha pour accroître ses capacités d'intervention dans les affaires de la République : «Le prince fonde de grands espoirs sur son alliance avec la maison des Sapieha et il escompte devenir par là l'homme le mieux informé des affaires polonaises, et disposer du parti le plus puissant dans le royaume de Pologne ${ }^{20}$. » L'aide des Sapieha était particulièrement indispensable pour occuper le trône du duché de Courlande, dont le roi de Pologne était le suzerain. C'est pour

15. Polski Słownik Biograficzny, 30, 2, 1987, p. 10 ; Dom Sapieżynski [Le lignage des Sapieha] Varsovie, 1995, p. 418.

16. Emanuel Rostworowski, O polską koronę : Polityka Francji w latach 1725-1733 [Le trône de Pologne : La politique de la France en 1725-1733], Wrocław : Zakład Naukowy im. Ossolińskich, 1958, p. 55.

17. RGADA, f. 11 , op. 1 , d. 57, 1. 5-7.

18. Héritage des Radziwiłł (branche de Birże) qui, après la disparition de Bogusław Radziwiłł (1669), mort sans héritier mâle, échoit à sa fille Ludwika Karolina. Celle-ci épouse, en 1687, en secondes noces, le prince de Neuburg Karl-Philipp. La partie de l'héritage située dans le GDL comprenait la principauté de Słuck et de Kopyl, les comtés de Siebież et de Newel, ainsi que Kopyś, Romanowo, Bielica, Kojdanów, Birże, Zabłudów, etc. Les prétendants aux « possessions de Neuburg » étaient les Radziwiłł de Nieśwież, les Ogiński et surtout les plus proches parents des Radziwiłł de Birże, les Sapieha, qui, à partir de 1714, agissent de concert avec Ludwik Pociej.

19. Kosińska, « Początki kariery Stanisława Poniatowskiego... », p. 417.

20. Sbornik RIO, 15, (1875), p. 304 ; Gustav von Mardefeld au roi de Prusse, Saint-Pétersbourg, 26 janvier 1726: «Ich habe angemerket, dass der Fürst von der Allianz mit dem Hause Sapieha sich sonderlich fête machet, und affectiret, in denen in polnischen Affairen mehr Lumières als andere und eine stärkere Partei im Königreich Polen zu haben. » 
discuter des termes de cette union que Jan Kazimierz, Antoni Kazimierz et Piotr Sapieha arrivent avec leur suite à Saint-Pétersbourg le 2 mars $1726^{21}$.

Le rival de Menšikov pour la principauté de Courlande est le fils illégitime d'Auguste II, Maurice de Saxe. Le couronnement de ce dernier comme prince de Courlande signifierait, entre autres, un renforcement de la dynastie saxonne des Wettin au sein de la République, ce qui n'est pas pour plaire à l'opposition antimonarchique dont les Sapieha font partie. Mais les Sapieha sont les seuls à soutenir Menšikov, le reste de l'opposition antimonarchique voyant d'un mauvais œil le renforcement de l'influence russe en Courlande.

Reçus à Saint-Pétersbourg en champions de l'opposition à Auguste II, les Sapieha sont accueillis avec la plus grande cordialité ${ }^{22}$. En quête de soutien russe pour l'opposition antimonarchique, Jan Kazimierz soumet à Catherine $\mathrm{I}^{\text {re }}$ un mémoire dans lequel Auguste II est accusé de violer les lois de la République et de s'efforcer de proclamer son fils héritier du trône ${ }^{23}$. Le séjour de Sapieha en Russie ne modifie pas son point de vue, et c'est l'impression qu'il produit sur l'ambassadeur de France, Jacques de Campredon :

M. Urbanowitz [...] et le comte Sapieha [...] me paraissent fort sincèrement attachés à la personne et aux intérêts du roi Stanislas. Ils m'ont paru même vouloir s'employer de tout leur pouvoir à traverser [empêcher] l'alliance de cette cour-ci avec celle de Vienne. ${ }^{24}$

Ces initiatives de Sapieha sont caractéristiques de l'opposition antimonarchique du GDL, qui entretenait des relations à la fois avec la Russie et avec la France, qui pourtant s'affrontaient sur la scène internationale.

Le principal, dans les relations entre Sapieha et Menšikov, reste cependant la question du duché de Courlande. La veille même du départ de Jan Kazimierz de Saint-Pétersbourg, le 2 avril 1726, il écrit à l'ambassadeur russe à Varsovie, Grigorij Dolgorukij, ainsi qu'à Mihail Bestužev à Mitau (ou : Mitawa, act. Jelgava) pour leur demander à tous deux de soutenir sa candidature à l'élection du duc de Courlande $^{25}$. Tous les efforts de Menšikov sont pourtant vains car, le 18 juin 1726, c'est Maurice de Saxe qui est élu duc de Courlande par la Diète unanime. Tout aussi improductif est le séjour de Menšikov à Mitau de la mi-juin à début juillet 1726. En dépit des menaces, les Courlandais refusent de soutenir sa candidature et restent

21. Wojciech Kriegseisen, « Diariusz Petersburskiej podróży Jana i Piotra Sapiehów z 1726 roku [Journal du voyage à Saint-Pétersbourg de Jan et Piotr Sapieha] », Wiek Oświecenia, 9, 1993, p. 57.

22. Rostworowski, O polską koronę..., p. 81.

23. Ibid., p. 55 ; Polski Słownik Biograficzny, p. 10 ; Dom Sapieżynski, p. 419.

24. Sbornik RIO, 64, SPb., 1888, p. 288 ; Campredon au comte de Morville, Pétersbourg, 9 mars 1726 .

25. Nikolaj Ivanovič Pavlenko, Menšikov : poluderžavnyj vlastelin [Menšikov, le potentat à demi-souverain], M., 1999, p. 251. 
fidèles à Maurice de Saxe. Les Sapieha, cependant, suivent attentivement la situation. Ils conseillent d'abord à Menšikov de contester l'élection devant le tribunal du GDL, promettant le soutien des magnats hostiles au roi et de Kazimierz Ogiński, maréchal [c'est-à-dire président] du tribunal (Marszałek trybunałski) et voïvode de Troki ; en échange, les Sapieha demandent à la cour du tsar d'accorder sa protection à Kazimierz Ogiński ${ }^{26}$. Leur appui arrive à point nommé et c'est sans aucun doute l'action des Sapieha qui dissuade Maurice de Saxe de faire valider son élection par le tribunal du GDL ${ }^{27}$. Le soutien apporté à Maurice par le grand hetman Ludwik Pociej, un allié de longue date des Russes, ne peut que renforcer la position des Sapieha aux yeux de Menšikov, puisque, dans le GDL, celui-ci ne peut désormais compter que sur eux ${ }^{28}$.

Jan Kazimierz apporte son appui à Menšikov à Saint-Pétersbourg aussi, où il discute avec le duc de Holstein de la possibilité pour Menšikov de devenir duc de Courlande $^{29}$. Sitôt informé par Sapieha, le duc de Holstein s'empresse de transmettre l'information à Catherine $\mathrm{I}^{\mathrm{re}}$, qui rejette le plan sans appe $\mathrm{l}^{30}$.

Comme on le voit, les Sapieha, non contents de nuire aux ambitions de Maurice de Saxe sur le duché de Courlande, soutiennent tant en Russie que dans le GDL la candidature de Menšikov. Leur but était d'empêcher la dynastie des Wettin de se renforcer, mais aussi d'asseoir solidement, grâce à la protection russe, leur propre position dans la République. Le 22 mars 1726, Jan Kazimierz reçoit de l'impératrice la dignité de feld-maréchal assortie d'une pension annuelle de 120000 «zlotys de Moscou $»^{31}$. Jean Lefort, ambassadeur de Saxe à Saint-Pétersbourg, considère que cette nomination « lui procurera de nouveau les grâces de Votre Majesté [Auguste II] par l'intercession de S.M. la Czarine et du duc de Holstein $»^{32}$. Menšikov tente des démarches analogues auprès d'Auguste II $^{33}$. C'est à cette époque qu'apparaissent des bruits invraisemblables : Catherine $\mathrm{I}^{\mathrm{re}}$ aurait caressé le projet de faire Piotr

26. RGADA, f. 198, op. 1, d. 920, lettre de Jerzy Felicjan Sapieha à Aleksandr Menšikov, Wilno, 26.05.1726,1.183.

27. Pavlenko, Menšikov..., p. 257.

28. Sbornik RIO, 3 (1868), p. 448-449.

29. Andrej Macuk, « Rol’ Jana Sapegi v obščestvenno-političeskih processah v Reči Pospolitoj i Velikogo kniažestva Litovskogo v pervoj polovine XVIII veka » [« Le rôle de Jan Sapieha dans la vie politique de la République et du Grand-duché de Lituanie dans la première moitié du XVIII ${ }^{\mathrm{e}}$ siècle »] », in Victoria, Gloria, Fama, SPb. : IVIMAiVs, 2003, p. 44.

30. Sbornik RIO, 15 (1875), p. 310.

31. Bibliothèque de 1'université de Lviv, fonds des Bavarovskij, $n^{\circ}$ 50472, f. 10 ; Jurij Nikolaevič Bespjatyh, Inostrannye istočniki po istorii Rossii pervoj četverti XVIII veka [Les sources étrangères de l'histoire de la Russie du premier quart du XVII' siècle], SPb. : Blic, 1998, p. 202 ; AGAD (Archiwum Główne akt dawnych - Archives centrales des actes anciens), Archiwum Rosji [Archives de Russie], LV/18, lettre de Antoni Kazimierz Sapieha à Jerzy Felicjan Sapieha, 05.03.1726, Saint-Pétersbourg, k. 56-58.

32. Sbornik RIO, 3 (1868), p.436, d. 119, Lefort au roi de Pologne et de Saxe, Saint-Pétersbourg, 26 mars 1726.

33. RGADA, f. 198, d. 171,1.1-2. 
Sapieha duc de Courlande et Jan Kazimierz roi de Pologne ${ }^{34}$. L'ambassadeur de Prusse, Gustav Mardefeld mentionnera plus tard cette seconde partie du plan, mais en attribuant sa paternité à Menšikov, dont telle aurait été l'intention, sitôt devenu tsar de Russie ${ }^{35}$. Catherine Ire , rompant les fiançailles de Marija Menšikova avec Piotr Sapieha, fait de ce dernier son favori, lui destinant comme épouse sa parente Sof ${ }^{\prime}$ ja Skavronskaja ${ }^{36}$. Piotr reçoit une pelisse de zibeline et une somme de 6000 roubles, son père une pelisse et 1800 ducats $^{37}$. De cette influence croissante des Sapieha en Russie témoigne la diffusion de la rumeur selon laquelle en cas de mort d'Auguste II, l'impératrice fournira à Jan Kazimierz 20000 soldats pour occuper le trône de Pologne ${ }^{38}$. En attendant, on projetait d'offrir à Jan Kazimierz le commandement du corps auxiliaire de l'armée russe pour venir en aide à l'Autriche dans le Hanovre. Mais l'opposition du corps des officiers généraux russes fit avorter le projet $^{39}$.

Les rumeurs qui courent pendant toute cette période ne suffisent pas à attester les ambitions royales de Jan Kazimierz. Lui-même semble considérer Stanisław Leszczyński comme le futur roi. Quant à ces rumeurs sur sa candidature au trône de Pologne, elles sont révélatrices : les élites au pouvoir en Russie continuaient de voir en lui un magnat du GDL, non un grand de Russie. Il n'est pas exclu que la candidature de Sapieha, partisan notoire de la Russie, ait été envisagée à SaintPétersbourg, où l'on était très mécontent de la politique d'Auguste II, et hostile aussi bien à une instauration durable de la dynastie des Wettin qu'à l'élection au trône de Leszczyński.

La position de Piotr et de Jan Kazimierz à la cour de Russie n'est pas affectée, non plus, par la mort de Catherine $\mathrm{I}^{\mathrm{re} 40}$. Le cadet, qui s'est tenu aux côtés de l'impératrice jusque dans ses derniers moments, fait partie des trois nouveaux chambellans nommés par Pierre $\mathrm{II}^{41}$. Et l'aîné est présent à la session du Conseil suprême privé où est lue l'instruction de Catherine $\mathrm{I}^{\mathrm{re}}$ quant à la succession au trône ${ }^{42}$. L'ambassadeur de Saxe Jean Lefort décrit la position des Sapieha à la cour de Russie avec justesse :

Quant à ce qui regarde le feld-maréchal Sapieha et son fils, il ne semble pas que les changements arrivés en aient produit aucun dans leur situation. Le fils est fort aimé du Czar, qui l'a demandé lui-même pour rester près de Sa personne, dont

34. Sbornik RIO, 24 (1878), p. 515, 521, 527, 532, 543.

35. Sbornik RIO, 15 (1875), p. 323.

36. Sbornik RIO, 26 (1879), p. 83.

37. Sbornik RIO, 3 (1868), p. 464.

38. Sbornik RIO, 64 (1888), p. 543.

39. Polski Słownik Biograficzny, p. 10 ; Dom Sapieżynski, p. 419.

40. Sbornik RIO, 3 (1868), p. 473.

41. Ibid., p. 471.

42. S.M. Solov'ev, vol. X, t. XIX-XX, M., 1993, p. 79. 
il est actuellement le premier chambellan. Le père s'est fait estimer parce qu'il n'est entré en aucune affaire et qu'il n'a pas abusé de son crédit. ${ }^{43}$

On voit ici ce que le renforcement de la position de Jan Kazimierz doit à sa neutralité dans les luttes politiques intérieures de la Russie. Ce qui ne l'empêche pas du reste de montrer, dans le même temps, une grande activité dans les luttes politiques internes de la République.

\section{Sapieha et l'échec de l'alliance russe}

Jouissant d'une influence exclusive sur le nouveau tsar, Menšikov décide de lui faire épouser sa fille aînée Marija, ancienne fiancée de Piotr Sapieha ${ }^{44}$. Ce qui se produit « de participation avec le feld-maréchal [Sapieha] ». Lequel s'est sans doute laissé fléchir par un certain nombre de considérations : l'attente d'un « dédommagement » et le désir de ne pas interférer avec les projets de Menšikov, dont le feldmaréchal comprenait fort bien la situation instable.

Les Sapieha parviennent à éviter la disgrâce qui frappe sous Pierre II leur ancien protecteur Menšikov. Qui plus est, Jan Kazimierz est, avec Aleksej Dolgorukij, du dîner à l'issue duquel Menšikov est assigné à résidence ${ }^{45}$. La présence de Jan Kazimierz auprès du tsar à ce moment montre que, s'il n'a pas pris l'initiative de l'arrestation de Menšikov, comme Dolgorukij, du moins il ne la désapprouve pas. Du reste cette arrestation renforce beaucoup plus qu'elle n'affaiblit les positions des Sapieha. Pendant quelque temps, Piotr Sapieha fait même partie de l'entourage le plus proche du souverain russe et, avec l'appui de ce dernier, épouse Sof' ja Skavronskaja ${ }^{46}$ - ce qui établit une relation de parenté entre les Sapieha et l'élite dirigeante de Russie. Quant à Jan Kazimierz, il prend part au conseil de famille impérial, aux sessions du Conseil suprême privé, du Sénat et du Saint-Synode, sans oublier le couronnement de Pierre II. On prépare sa nomination comme généralgouverneur de Saint-Pétersbourg ${ }^{47}$. En Russie, Jan Kazimierz et Piotr sont ainsi parvenus à s'intégrer dans l'élite dirigeante russe, ce qui, par contrecoup, rehausse encore, aux yeux des gens de Saint-Pétersbourg, l'importance du clan dans la vie politique du GDL. À la fin de 1727 le bruit se répand que l'un des alliés des Sapieha à la cour de Russie, Pavel Jagužinskij, cherche à contracter alliance avec ces derniers en mariant sa fille à l'un d'eux ${ }^{48}$.

43. Sbornik RIO, 3 (1868), p. 473, d. 151, Lefort au roi, Saint-Pétersbourg, 24 mai 1727.

44. Ibid., p. 478.

45. Ibid., p. 494.

46. RGADA, f. 11, op. 1, d. 57, 1. 3-4, 12 ; f. 198, op. 1, d. 171,1. 1-2.

47. Sapiehowie : Materjały historyczno-genealogiczne i majatkowe [Les Sapieha : documents relatifs à l'histoire, la généalogie, la situation de fortune du lignage], vol. III, SPb., 1890, p. 136 ; Bespjatyh, Inostrannye istočniki...,p. 202 ; Polski Słownik Biograficzny, p. 10.

48. Sbornik RIO, 3 (1868), p. 504. 
Aussi rapide que leur ascension, et aussi inattendue, fut la chute de leur influence à la cour de Russie. Dès octobre 1727, ils se heurtent à l'opposition du feld-maréchal Mihail Golicyn, qui a lui aussi des vues sur la charge de général-gouverneur de Saint-Pétersbourg ${ }^{49}$. Pierre II tranche cependant le conflit au profit des Sapieha : « Il ne paraît pas que les Galizine aient les rieurs de leur côté. [...] Il [le feld-maréchal Golicyn] a recherché avec soin le gouvernement de Pétersbourg, mais le Czar est resté ferme, que Sapiéha l'aurait. » Jan Kazimierz doit une grande partie de sa victoire à l'appui d'Osterman. « Il est vrai que le baron Ostermann y a beaucoup contribué, qui est fort ami de Sapiéha, ainsi que de Jagouzinski ${ }^{50}$. » À la fin de l'année 1727, cependant, on apprend la nomination de Jan Kazimierz, et simultanément qu'un étranger ne peut occuper cette charge ${ }^{51}$. Du reste, le centre d'intérêt principal des Sapieha se situe toujours dans le GDL, où Jan Kazimierz repart dès 1728. Parmi les raisons invoquées pour ce départ, on cite le retour sur ses terres de Grande Pologne pour cause de santé ${ }^{52}$, mais aussi une mission dont l'aurait chargé Pierre II : « écouter et rapporter ce qui se passe en Pologne concernant la succession au trône $»^{53}$. On fait aussi état de la rivalité qui l'oppose au puissant lignage des Golicyn pour le poste de général-gouverneur de Saint-Pétersbourg. Il semble donc bien que le départ de Jan Kazimierz pour le GDL ait été causé, entre autres, par l'affaiblissement de son influence à la cour de Russie.

On ne peut pas dire du reste que son activité dans les diétines d'élection des nonces $^{54}$ au printemps 1728 ait été couronnée d'un grand succès. Il aurait voulu faire élire son cousin issu de germains, Antoni Kazimierz, comme nonce/député à la Diète, dans l'espoir d'en faire un jour le maréchal de la Diète (Marszałek Sejmu), mais n'y parvient pas ${ }^{55}$. On lui adjoint le major Krzysztof (Christoph) von Lieven, qui doit le surveiller car on le soupçonne de « se laisser guider davantage par son penchant pour Stanisław que par les intérêts de la Russie, qui s'oppose à ce dernier ${ }^{56}$. Ces soupçons se vérifiant, on décide de rappeler Jan Kazimierz d'urgence. Privé de perspectives en Russie, Sapieha rompt avec ses anciens alliés et reste à la Diète de 1729 à Grodno. Il y entame une collaboration avec l'ambassadeur de France Antoine-Félix de Monti, ainsi qu'une correspondance avec le Cardinal Fleury ${ }^{57}$, alors à la tête des affaires en France. On prévoit un déménagement à

49. Ibid., p. 503.

50. Ibid., p. 504, d. 179, Lefort au roi, Saint-Pétersbourg, 4 novembre 1727.

51. Kriegseisen, « Diariusz Petersburskiej... », p. 62 ; Sbornik RIO, 64 (1888), p. 513.

52. Sapiehowie : Materjały..., p. 136.

53. Rostworowski, O polską koronę..., p. 149.

54. Les assemblées provinciales, ou « diétines » (sing. sejmik), préparaient le travail de la Diète (Sejm) et y élisaient leurs députés, ou « nonces » (sing. posef) (NdT)

55. Polski Słownik Biograficzny, p. 10 ; Dom Sapieżynski, p. 419.

56. Rostworowski, O polską koronę..., p. 149.

57. Sbornik RIO, 25 (1878), p. 279. 
Paris de Piotr Sapieha ${ }^{58}$, qui doit renforcer ce nouveau partenariat. Cette volte-face où Paris remplace Saint-Pétersbourg signifie bien que Jan Kazimierz, persuadé de l'impossibilité d'accomplir ses desseins avec l'appui de la Russie, s'efforce désormais d'obtenir ailleurs l'aide qui lui est indispensable : auprès de la France, l'adversaire de la Russie. En même temps, il se dit que la Russie ne s'opposera pas à l'élection de Leszczyński si ce dernier s'engage à ne pas agir de conserve avec la Suède contre les intérêts russes. Il n'est pas sans intérêt de constater que parallèlement le plénipotentiaire russe Mihail Bestužev échange des propos analogues avec Antoine-Félix de Monti ${ }^{59}$. Ce qui suggère que lorsque Jan Kazimierz fait état d'un possible soutien de Stanisław Leszczyński par la Russie, il reflète la position d'un groupe bien précis de dignitaires russes.

Après l'avènement de l'impératrice Anna, Jan Kazimierz tente de recouvrer son influence à Saint-Pétersbourg : il achète Osterman pour qu'il défende ses intérêts. Difficile d'estimer le résultat de ces manœuvres, car c'est à ce moment que Jan Kazimierz meurt ${ }^{60}$.

Ainsi nous avons vu comment, dans la vision du monde d'un lignage de magnats, en peu de temps l'image de la Russie se transforme : la puissance ennemie, qui soutient les adversaires des Sapieha à l'intérieur de la République, devient une alliée précieuse contre le roi Auguste II. Cette évolution doit beaucoup aux contacts dont disposent les Sapieha au sein de l'élite dirigeante russe, en premier lieu avec Aleksandr Menšikov. Pendant un temps, leurs intérêts cö̈ncident. Les Sapieha soutiennent les prétentions de Menšikov au duché de Courlande, afin de limiter la puissance des Wettin dans la République. Inversement, la protection de Menšikov permet aux Sapieha d'affermir leurs positions à la cour de Russie, et de bénéficier de l'appui de leurs alliés russes pour leurs combinaisons dans le GDL. L'union des Sapieha et des Menšikov aurait dû être renforcée par le mariage de Piotr Sapieha et de Marija Menšikova, mais la noce n'a pas lieu et l'alliance politique de Menšikov et des Sapieha tourne court. Les seconds, sous Pierre II, parviennent malgré tout à maintenir leur crédit à la cour de Russie, par le mariage de Piotr Sapieha avec Sof'ja Skavronskaja cette fois, sans perdre le soutien de la Russie dans les luttes intérieures du GDL. Les Sapieha voient également en la Russie une force capable de favoriser l'accession au trône de la République de Stanisław Leszczyński. Cependant leurs positions à Saint-Pétersbourg se révèlent fragiles et, comprenant qu'il leur sera impossible de réaliser leurs plans avec l'appui la Russie, ils lui préfèrent la protection de la France.

Après la mort de Jan Kazimierz le clan Sapieha n'entretient plus les mêmes relations avec la cour de Russie, mais reste toutefois en contact direct avec l'impératrice ${ }^{61}$. Les Ogiński, alliés des Sapieha, s'y montrent les plus actifs. C'est

58. Macuk, « Rol’ Jana Sapegi... », p. 45.

59. Rostworowski, O polską koronę..., p. 149.

60. Ibid., p. 150.

61. Macuk, « Rol’ Jana Sapegi... », p. 45. 
ainsi que Kazimierz Ogiński, voïvode de Wilno, entretient une correspondance nourrie avec elle, sollicitant sa protection pour son lignage ${ }^{62}$. Sa femme Eleonora fait de même, mettant à profit les relations que sa fille Helena a nouées avec l'impératrice à l'époque où celle-ci était encore duchesse de Courlande ${ }^{63}$. Au printemps 1731, Helena va même passer quelque temps à Saint-Pétersbourg ${ }^{64}$.

Cependant, parmi les magnats du GDL hostiles au roi, on commence à placer ses espoirs non plus dans une aide de la Russie, mais dans celle de la France. Cela s'explique à la fois par l'affaiblissement des relations avec la Russie après la mort de Jan Kazimierz Sapieha et de Stanisław Denhoff en 1728, de Ludwik Pociej en 1730 , et par l'action décidée que mène dans la République l'ambassadeur de France Antoine-Félix de Monti en faveur de Stanisław Leszczyński. Symétriquement, les anciens partisans de Leszczyński en Russie rompent avec l'opposition antimonarchique du GDL, restée fidèle à son candidat initial Leszczyński, ce qui achève de transformer le paysage politique.

La perception de la société russe par ces anciens partisans lituaniens de Stanisław Leszczyński méritait une étude approfondie. Elle nous a permis de comprendre quelques-unes des raisons de leur passage au service de la Russie et l'établissement de relations étroites, parfois d'alliances matrimoniales, avec les cercles dirigeants russes. En explorant leurs engagements de toutes sortes dans les affaires intérieures de la République, leur mode d'intégration dans l'élite dirigeante du GDL et celle de Russie, leurs relations avec les diplomates accrédités auprès de la République, de la Russie ou de la Saxe, nous avons pu mettre au jour leurs contradictions et retracer la genèse d'une idée paradoxale s'il en fut : favoriser l'avènement de Stanisław Leszczyński avec l'aide simultanée de la Russie et de la France.

\section{Urbanowicz et le retour à l'alliance russe}

Jusqu'à présent ce sont des personnalités telles que Krzysztof Urbanowicz, Krzysztof et Jerzy Lieven, Franciszek Darewski qui ont surtout retenu l'attention des historiens. Nous nous limiterons ici au premier, dans la mesure où les Lieven et Darewski se sont contentés de reproduire les méandres de son évolution : Russie rivale, Russie possible alliée de Leszczyński, enfin Russie alliée, mais hostile à Leszczyński.

Urbanowicz s'était couvert de gloire en combattant aux côtés des Suédois pendant la seconde Guerre du Nord. À son retour, il commence par soutenir le parti de la cour et collabore avec son chef, qui a commandé, lui aussi, dans les armées

62. RGADA, f. 12, op. 1, d. 63, lettre de Kazimierz Ogiński à Anna Ioannovna, Wilno, 14.07.1730, f. 1-2.

63. Ibid., d. 64, lettre de Eleonora Ogińska à Anna Ioannovna, s.1. s.d., f. 1-2.

64. Teresa Zielińska, « Ogiński Kazimierz », Polski Słownik Biograficzny, 23, 3, 1978, p. 616 . 
suédoises, Stanisław Poniatowski ${ }^{65}$. Cependant il passe rapidement du côté de l'opposition antimonarchique. Jan Kazimierz Sapieha le recommande pour servir en Russie : il serait utile à Pierre $\mathrm{I}^{\text {er }}$ " dans l'intérêt de la Pologne ${ }^{66}$. Leszczyński lui aussi se réjouit de l'entrée de son ancien compagnon d'armes au service de la Russie : «Rien ne pourrait m'être plus utile que de te savoir en ce service et à cet endroit, où tu peux faire beaucoup de bien. [...] Il ne pouvait rien advenir de mieux compte tenu de la conjoncture actuelle. ${ }^{67} \gg$ Leszczyński place de grands espoirs dans l'aide de Menšikov et c'est pourquoi il écrit à Urbanowicz : « Je vous prie de saluer pour moi le prince Menšikov et de lui recommander mes intérêts. ${ }^{68}$ » C'est Sapieha qui recommande Urbanowicz à Menšikov, non sans souligner qu'il lui est apparenté par sa femme ${ }^{69}$. Urbanowicz ne se contente pas de figurer parmi les familiers de Menšikov, il est aussi son homme de confiance dans un certain nombre d'affaires concernant la République. Il l'assiste également dans les négociations avec Jan Kazimierz au sujet du mariage de son fils Piotr avec Marija Menšikova ${ }^{70}$. C'est à l'initiative de Menšikov qu'au début de 1726 Urbanowicz se rend dans le GDL pour soutenir l'opposition antimonarchique contre le projet d'Auguste II de faire élire son fils comme roi de Pologne-Lituanie ${ }^{71}$. On retrouve Urbanowicz dans les projets de Menšikov de devenir duc de Courlande avec l'aide des Sapieha et autres alliés du GDL. Il est tout à fait certain que c'est à travers lui que Menšikov demeure en relation, au cours de toute cette affaire, avec Jan Plater ${ }^{72}$ et Paweł Sanguszko, le maréchal de la cour (Marszałek nadworny) du GDL ${ }^{73}$. En échange, Urbanowicz aide les Sapieha à réaliser leurs projets en Russie ${ }^{74}$. Il est vrai que le rapprochement entre son protecteur russe Menšikov et les Sapieha sert ses intérêts, en lui offrant la possibilité de se montrer utile à la fois au premier et aux seconds.

Ceci ne l'empêche pas du reste de servir, au côté de Jan Kazimierz, les intérêts de Stanisław Leszczyński. Il collabore pour ce faire avec le chargé d'affaires français en Russie, Magnan, et avec de Monti, l'ambassadeur de France auprès de la

65. Kosińska, « Początki kariery Stanisława Poniatowskiego... », p. 417.

66. RGADA, f. 198, op. 1, d. 920, lettre de Jan Kazimierz Sapieha à Aleksandr Menšikov, Rawicz, 25.11.1724, f. 104.

67. Rostworowski, O polską koronę..., p. 53.

68. Ibid.

69. RGADA, f. 198, op. 1, d. 920, lettre de Jan Kazimierz Sapieha à Aleksandr Menšikov, Rawicz, 25.11.1724, f. 104.

70. Ibid., f. 156, 157.

71. Sbornik RIO, 15 (1875), p. 302.

72. Lietuvos Mokslų Akademijos centrinès bibliotekos Rankraščių skyrius [Bibliothèque centrale de l'Académie des sciences de Lituanie, département des manuscrits], f. 198, b. 40, 1. 2 .

73. Archiwum Państwowe na Wawelu, Archiwum Sanguszków [Archives d'État au château de Wawel (Cracovie), Archives des Sanguszko], teki korespondencji [Correspondance, portefeuille] 6,k. 950.

74. AGAD, Archiwum Rosji, LV/18 : Antoni Kazimierz Sapieha à Jerzy Felicjan Sapieha, 05.03.1726, Saint-Pétersbourg, k. 58. 
République. Par l'intermédiaire de ce dernier, Urbanowicz entretient une correspondance avec Leszczyński lui-même, ainsi qu'avec le chef de la diplomatie française, Germain-Louis Chauvelin - lequel le considère comme « un homme sage, influent et prudent $\gg^{75}$. De cette façon, l'ascension rapide d'Urbanowicz en Russie le transforme peu à peu de simple intermédiaire entre l'élite politique russe et les magnats du GDL (au premier rang desquels les Sapieha, il est vrai) en un acteur à part entière, de plus en plus indépendant. Son regard sur la Russie et sur l'élite politique russe a joué un rôle de premier plan dans l'attitude à l'égard de la Russie des anciens partisans de Leszczyński, et dans le passage de certains d'entre eux au service de ce pays. À la cour de Saint-Pétersbourg, Urbanowicz est partisan d'une alliance entre la Russie et la France. Il mène en son nom propre des négociations avec les magnats du GDL et les diplomates français, en promettant aux uns et aux autres le soutien de la cour de Russie. Ce rôle nouveau d'Urbanowicz devient particulièrement patent après le retour de Jan Kazimierz en Pologne-Lituanie.

Après la mort de Pierre II, tandis que s'affrontent les Dolgorukij et les Golicyn, Leszczyński compte sur le soutien à Saint-Pétersbourg d'Urbanowicz et de Darewski. Or Urbanowicz, grâce à ses contacts personnels au palais, n'apprécie pas la situation à la cour de Russie comme le font les autres partisans de Leszczyński. Un temps, il est tenté d'abandonner le service du tsar par suite de ses mauvaises relations avec Aleksej Dolgorukij. C'est d'ailleurs pourquoi, contrairement à d'autres partisans de Leszczyński, il se réjouit lorsque la tentative de limiter les pouvoirs du souverain russe échoue ${ }^{76}$.

Or sous le règne d'Anna Ioannovna les relations personnelles des magnats avec la tsarine prennent une importance nouvelle. C'est Urbanowicz qui, ayant étudié la personnalité de la souveraine, suggère à Antoni Potocki, en tant que parent de la tsarine, de venir à Saint-Pétersbourg. Il conseille en même temps à Leszczyński d'envoyer à l'impératrice la lettre de Pierre ${ }^{\text {er }}$ lui promettant l'aide de la Russie contre Auguste $\mathrm{II}^{77}$. Il espère ainsi la convaincre de soutenir la candidature de Leszczyński au trône de Pologne-Lituanie. L'intéressé, au demeurant, prie luimême Urbanowicz de défendre ses intérêts auprès d'elle ${ }^{78}$.

À la cour impériale, Urbanowicz s'est rapproché de la faction nobiliaire des « vieux Russes ${ }^{79}$ » (starorusy). Il est à même par conséquent de renseigner ses correspondants sur la position des «vieux Russes », et avant tout celle de Jagužinskij, qui a toute confiance en lui, sur les principales questions de politique étrangère.

75. Rostworowski, O polską koronę..., p. 152.

76. Ibid., p. 153-154.

77. Ibid., p. 225-226, 228.

78. Aleksander Przeździecki, « Krzysztof Urbanowicz, starosta hubski : Obrazek historyczny z listów własnoręcznych króla Stanisława Leszczyńskiego skreślony [Krzysztof Urbanowicz, starosta de Hub : tableau historique peint d'après les lettres de la main du roi Stanisław] », Biblioteka Warszawska, 63, 1856, p. 16.

79. C'est le sobriquet donné aux favoris et dignitaires des règnes précédents (ceux de Pierre $\mathrm{I}^{\mathrm{er}}$, Catherine Ire, Pierre II), qui s'opposent à l'ascension des « Allemands », venus dans les bagages de l'ancienne duchesse de Courlande (NdT) 
Parlant au nom des partisans de Leszczyński, Urbanowicz se décide à passer un accord avec Jagužinskij : la Russie doit s'opposer par tous les moyens au dessein d'Auguste II de porter son fils sur le trône. Aussi les partisans de Leszczyński en Lituanie éprouvent-ils de la sympathie pour les « vieux Russes » dans leur lutte contre les «Allemands ». Ils ressentent la déroute finale des vieux Russes comme la défaite d'un allié, et cela ne fait que renforcer leur hostilité aux « Allemands ».

Mais l'évolution d'Urbanowicz ne s'arrête pas là, et il se rallie bientôt à l'idée d'une alliance entre la Russie et l'Autriche. Magnan, le chargé d'affaires français en Russie, remarque qu'Urbanowicz ne vient plus le voir, mais rend souvent visite à l'ambassadeur autrichien à Moscou. Urbanowicz se plaint de l'avarice de Stanisław Leszczyński, qui a causé la perte d'un appui aussi actif que Darewski ${ }^{80}$. Il finit par cesser tout contact avec les représentants de la France, et montre aux Russes et aux Autrichiens une lettre de Leszczyński où celui-ci accorde son soutien à Stanisław Poniatowski, chef de file du groupe promonarchique de la République, qui voudrait être nommé grand hetman de la Couronne. C'est encore Urbanowicz qui est soupçonné d'avoir dévoilé le secret de la correspondance entre Leszczyński et Jagužinskij. Sans que l'affaire puisse être tirée au clair : Urbanowicz, ivre, se noie dans la Moskova en septembre $1731^{81}$. De sorte qu'à la fin de sa vie, Urbanowicz rompt avec l'opposition antimonarchique du GDL, alors liée à la France, pour se mettre à agir exclusivement dans l'intérêt de la Russie.

\section{Conclusion}

L'étude des contacts entre divers lignages de magnats et les représentants de l'élite politique russe nous a permis de décrire l'idée que se fait tel ou tel clan du rôle de la Russie dans ses relations avec le grand-duché de Lituanie et avec les autorités de la République. La position des magnats a évolué en fonction de leur propre situation dans le GDL et des conflits internes du grand-duché. Autre facteur très important, les liens de parenté ou d'alliance qui unissent de nombreux représentants des élites dirigeantes russes à ceux de la République.

L'opposition de la noblesse, emmenée par les magnats, aux efforts faits par Auguste II pour renforcer le pouvoir monarchique a trouvé dans la Russie un allié précieux. C'est grâce à elle que les hetmans du GDL, Ludwik Pociej et Stanisław Denhoff, ont obtenu le retour sous leur autorité des troupes « sur le modèle étranger ». Le clan des Sapieha va plus loin : avec l'aide de Menšikov, ils s'intègrent dans l'élite politique russe, tout en continuant à soutenir à Moscou la cause de Stanisław Leszczyński.

Cependant l'opposition antimonarchique se divise sur la question du duché de Courlande. Tandis que Ludwik Pociej soutient, contre la Russie, la candidature de Maurice de Saxe, les Sapieha appuient celle d'Aleksandr Menšikov. Quant à la

80. Rostworowski, O polską koronę..., p. 233.

81. Ibid., p. 216, 234-235. 
noblesse du rang, elle rejette les deux candidats et exige le rattachement du duché à la République. Une fois de plus, les magnats et la noblesse se montrent incapables d'agir de concert, comme de s'entendre sur le rôle à donner à la Russie.

La candidature de Stanisław Leszczyński au trône de Pologne-Lituanie, vivement soutenue par la France, est une nouvelle pomme de discorde. Les Sapieha quittent Saint-Pétersbourg et rompent leurs relations avec l'élite politique russe, pour rechercher l'appui de la France. Krzysztof Urbanowicz, à l'inverse, part de l'idée que l'aide de la Russie est indispensable pour l'élection de Stanisław Leszczyński. C'est d'abord dans ce but qu'il reste à Saint-Pétersbourg, mais il finit par servir les intérêts russes, ce qui l'amène à rompre avec les partisans de Leszczyński comme avec la France. C'est de celle-ci désormais que l'opposition antimonarchique du grand-duché de Lituanie attend le salut.

(traduit du russe par Stéphane Dudoignon)

Académie nationale des sciences de Biélorussie Institut d'histoire

amacuk@onet.eu 